Reporte de caso

\title{
Enfermedad de Erdheim-Chester: primer caso pediátrico reportado en Colombia
}

Luis Carlos Salazar ${ }^{1}$, Luz Ángela Moreno ${ }^{1,2}$, Lina Eugenia Jaramillo ${ }^{3,4}$, Edgar Vladimir Cabrera ${ }^{5}$

${ }^{1}$ Departamento de Radiología e Imágenes Diagnósticas, Facultad de Medicina, Universidad Nacional de Colombia, Bogotá, D.C., Colombia

2 Unidad Funcional de Imágenes Diagnósticas, Fundación Hospital de La Misericordia, Bogotá, D.C., Colombia

${ }^{3}$ Departamento de Patología, Facultad de Medicina, Universidad Nacional de Colombia, Bogotá, D.C., Colombia

${ }^{4}$ Laboratorio de Patología, Fundación Hospital de La Misericordia, Bogotá, D.C., Colombia

${ }^{5}$ Servicio de Oncohematología Pediátrica, Fundación Hospital de La Misericordia, Bogotá, D.C., Colombia

La enfermedad de Erdheim-Chester es una condición extremadamente rara en la edad pediátrica. Se presenta el caso de una niña de 12 años con diagnóstico histológico y radiológico de enfermedad de Erdheim-Chester multisistémica y mutación en el gen BRAF, que requirió tratamiento con dabrafenib.

Hasta el momento, se han reportado 22 casos pediátricos en el mundo y este es el segundo en Latinoamérica. Se observó el hallazgo radiológico denominado signo oscuro paraselar, descrito hasta ahora en pacientes con hipofisitis autoinmunitaria para diferenciarlos de aquellos con adenomas hipofisarios.

Este reporte contribuye a la literatura médica en dos aspectos fundamentales: las manifestaciones clínicas de la enfermedad y su diagnóstico en la población pediátrica.

Palabras clave: enfermedad de Erdheim-Chester; histiocitosis; pediatría; proteínas protooncogénicas B-raf; radiología; imagen por resonancia magnética.

\section{Erdheim-Chester disease: First pediatric case report in Colombia}

The Erdheim-Chester's disease is extremely rare in children. We present the case of a 12-year-old girl with histological and radiological diagnosis of this disease and mutation of the $B R A F$ gene, who developed multisystemic compromise requiring treatment with dabrafenib. We identified 22 reports of this condition among children worldwide and this is the second pediatric case in Latin America. Diagnostic imaging is critical to confirm Erdheim-Chester disease and for the surgical planning of the biopsy. Additionally, we identified the parasellar dark sign, which has previously been reported on lymphocytic hypophysitis.

This report contributes to the current practice as it shows the clinical presentation and the diagnostic workout of this disease in pediatrics.

Recibido: 17/07/2020

Aceptado: $17 / 06 / 2021$

Publicado: 17/06/2021

Citación:

Salazar LC, Moreno LÁ, Jaramillo LE, Cabrera

EV. Enfermedad de Erdheim-Chester: primer caso pediátrico reportado en Colombia. Biomédica. 2021;41:615-24.

https://doi.org/10.7705/biomedica.5651

\section{Correspondencia:}

Luis Carlos Salazar, Departamento de Radiología e Imágenes Diagnósticas, Facultad de Medicina Universidad Nacional de Colombia, Carrera 45 No 26-85, edificio 471, oficina 102, Bogotá, D.C., Colombia

Teléfono: (571) 316 5000, extensión 15109

Icsalazard@unal.edu.co

Contribución de los autores:

Todos los autores participaron en la obtención de los datos clínicos y de los estudios de extensión, el análisis de la información, la búsqueda de la bibliografía y la elaboración del manuscrito.

Financiación:

No se recibió ninguna.

Conflicto de intereses:

No se declaran conflictos de interés.
Keywords: Erdheim-Chester's disease; hypophysitis; pediatrics; B-Raf proto-oncogene proteins; magnetic resonance imaging.

La enfermedad de Erdheim-Chester hace parte del grupo de las histiocitosis, las cuales se caracterizan por ser trastornos sistémicos en los cuales hay una proliferación descontrolada de células derivadas del sistema mononuclear fagocítico (células dendríticas, monocitos y macrófagos), que infiltran diferentes órganos del cuerpo (1). Recibe su nombre gracias a la publicación realizada en 1930 por los patólogos Jakob Erdheim (Austria) y William Chester (Estados Unidos) (2). Clásicamente, se la describe como una histiocitosis de fagocitos mononucleares distintos a las células de Langerhans y pertenece al grupo II según la clasificación del Working Group of the Histiocyte Society de 1987 (3). En la clasificación de la Histiocyte Society de 2016 corresponde al grupo L, o grupo Langerhans, que reúne la histiocitosis de células de Langerhans, la histiocitosis de células indeterminadas, la enfermedad de Erdheim-Chester y la mixta, de histiocitosis de células de Langerhans más enfermedad de Erdheim-Chester (1).

A pesar de ser una histiocitosis, se considera una condición extremadamente infrecuente en la edad pediátrica, siendo más común en la adultez (4); es parte del grupo de las enfermedades raras, según la European 
Rare Disease Organization y la National Organization for Rare Disorders. En el 2014, el número total de casos reportados fue de 550, la gran mayoría de ellos diagnosticados en la última década, cifra que sigue aumentando debido al reconocimiento de la enfermedad (5).

Oskaya, et al., reportaron mutaciones en los genes BRAF en el $65 \%$ de los pacientes con esta condición y en el gen MAP2K1 en el $16 \%$. Estos hallazgos, junto con las mutaciones en otras proteínas cinasas activadas por mitógenos, sugerirían la naturaleza tumoral de la enfermedad y su clasificación como una neoplasia histiocitaria. Sin embargo, en la actualidad la enfermedad de Erdheim-Chester se considera una condición benigna de naturaleza inflamatoria (6).

El síntoma más frecuentemente descrito en esta enfermedad es el dolor óseo; asimismo, la diabetes insípida hace parte de su presentación habitual, tal como ocurre en la histiocitosis de células de Langerhans. La enfermedad de Erdheim-Chester puede cursar casi sin síntomas cuando el compromiso es predominantemente cutáneo u óseo, o ser sintomática, si predomina el compromiso del sistema nervioso central, cardiaco, retroperitoneal, órbitocraneo-facial, neuroendocrino, pulmonar o multisistémico (4).

El diagnóstico se hace con base en parámetros histológicos y radiológicos. Histológicamente, se caracteriza por la presencia de histiocitos cargados de lípidos con aspecto xantomatoso en un fondo de fibrosis. Cuando se ven células gigantes de Touton, la enfermedad es indistinguible del xantogranuloma juvenil, motivo por el cual los hallazgos clínicos y radiológicos son fundamentales para establecer el diagnóstico definitivo (4). Estas células son reactivas en la inmunohistoquímica para CD68, CD163 y el factor XIIla, y negativas para CD1a y langerina (CD207), en tanto que el marcador S100 tiene reactividad variable. En la ultraestructura, carecen de gránulos de Birbeck.

La enfermedad se caracteriza por un compromiso osteoesclerótico metafisiario y simétrico de los huesos largos, tanto en la tomografía como en la resonancia magnética, lo cual a menudo no se observa en las proyecciones radiográficas debido a su menor sensibilidad. Existe una fuerte captación metafisiaria en la gammagrafía ósea con Tc-99m. Sin embargo, el $4 \%$ de los pacientes con enfermedad de Erdheim-Chester no presenta el compromiso óseo radiológico clásico, por lo que en esos casos es necesario basarse en la histopatología y en otros órganos usualmente involucrados (4).

La enfermedad es de mal pronóstico, con una supervivencia de 32 meses en el $57 \%$ de los casos (7), y es de peor pronóstico cuando existe compromiso cardiaco o del sistema nervioso central. No obstante, la supervivencia ha aumentado con el advenimiento de los nuevos tratamientos. El medicamento de primera línea es el interferón alfa y la segunda línea incluye el vemurafenib, infliximab, anakinra o el dabrafenib $(8,9)$.

\section{Presentación del caso}

Se trata de una niña de 12 años que consultó por dolor abdominal, ictericia, coluria, acolia y prurito generalizado asociado con pérdida de peso ( $3 \mathrm{~kg}$ en un mes). Entre los antecedentes, se registró el nacimiento a término sin complicaciones, con bajo peso al nacer para la edad gestacional $(2.400$ $\mathrm{g}$ ), adecuado desarrollo psicomotor y buen rendimiento académico. En la consulta inicial, se evidenció baja talla para la edad (desviación estándar de $-3,58$ ) y ausencia de signos de desarrollo sexual secundario como 
pubarquia o telarquia. Además, la paciente refirió polidipsia, poliuria y dolor osteoarticular desde los primeros años de vida.

En el examen físico de ingreso, no se registró alteración de los signos vitales y la presión arterial era normal para la edad; se detectó ictericia, no había adenomegalias en el cuello, y el abdomen era blando y depresible, con leve dolor en el hipocondrio derecho. En el examen neurológico, no se encontró alteración de la esfera mental ni déficit cognitivo, el lenguaje era fluido, los reflejos oculomotores bilaterales estaban conservados y los movimientos oculares eran simétricos y sin restricción, aunque había disminución de la agudeza visual en el ojo izquierdo y nistagmo multidireccional con la mirada extrema derecha; no se registraba déficit motor o alteración de los reflejos musculotendinosos en las cuatro extremidades, había reflejo plantar neutro bilateral, sin signos cerebelosos ni alteraciones de la marcha.

En los exámenes de laboratorio no se encontraron alteraciones hematológicas, como coagulopatías, ni elevación de la concentración sérica del complemento; entre los reactantes de fase aguda, la velocidad de sedimentación globular (VSG) estaba elevada, en tanto que el valor de la proteína $C$ reactiva era normal. La paciente presentaba elevación del sodio sérico con densidad urinaria disminuida, pero sin alteración de la función renal. Se observó aumento de la gamma-glutamil transferasa (GGT), de la fosfatasa alcalina y de la bilirrubina conjugada; las concentraciones de alanino aminotransferasa (ALT) y de aspartato aminotransferasa (AST), eran normales. Se comprobó que había hipercolesterolemia y no hubo alteración de las proteínas séricas. No se observaron cambios de infección reciente en los exámenes específicos practicados. Se demostró panhipopituitarismo hipogonadotrófico con hipotiroidismo central e hipocortisolismo (cuadro 1).

Cuadro 1. Resultados de las pruebas de laboratorio

\begin{tabular}{|c|c|c|c|c|c|}
\hline \multicolumn{2}{|c|}{ Hematológicas } & \multicolumn{2}{|c|}{ Función hepática } & \multicolumn{2}{|c|}{ Perfil infeccioso } \\
\hline Prueba & Resultado & Prueba & Resultado & Prueba & Resultado \\
\hline Leucocitos & 10.730 por $\mathrm{ml}$ & ALT & $83,6 \mathrm{U} / \mathrm{L}$ & IgG toxoplasma & Negativo \\
\hline Hemoglobina & $11,5 \mathrm{~g} / \mathrm{dl}$ & AST & $98,8 \mathrm{U} / \mathrm{L}$ & IgM toxoplasma & Negativo \\
\hline Plaquetas & 261.000 por $\mathrm{mm}^{3}$ & $\mathrm{GGT}^{\star \star}$ & $724,2 \mathrm{U} / \mathrm{L}$ & IgG citomegalovirus & Positivo \\
\hline INR & 0,94 & LDH & $85 \mathrm{U} / \mathrm{L}$ & IgM citomegalovirus & Negativo \\
\hline C3 & $171 \mathrm{mg} / \mathrm{dl}$ & $\mathrm{FA}^{* \star}$ & $1.682 \mathrm{mg} / \mathrm{dl}$ & IgG Epstein-Barr & Positivo \\
\hline C4 & 27 mg/dl & Albúmina & $3,1 \mathrm{~g} / \mathrm{dl}$ & IgM Epstein-Barr & Negativo \\
\hline Glucosa & 84 mg/dl & Bilirrubina total** & $4,3 \mathrm{mg} / \mathrm{dl}$ & IgM HAV & Negativo \\
\hline Lactato & $1,2 \mathrm{mmol} / \mathrm{L}$ & Bilirrubina directa** & $4,2 \mathrm{mg} / \mathrm{dl}$ & $\mathrm{AgHBs}$ & Negativo \\
\hline VSG $^{* *}$ & $56 \mathrm{~mm} /$ hora & \multicolumn{2}{|c|}{$\begin{array}{c}\text { Función renal } \\
\end{array}$} & Anti-HCV & Negativo \\
\hline PCR & $16 \mathrm{mg} / \mathrm{L}$ & Prueba & Resultado & RPR & Negativo \\
\hline \multicolumn{2}{|c|}{ Electrolitos } & Creatinina & $0,34 \mathrm{mg} / \mathrm{dl}$ & \multicolumn{2}{|c|}{ Endocrinológicos } \\
\hline Prueba & Resultado & BUN & $5,2 \mathrm{mg} / \mathrm{dl}$ & Prueba & Resultado \\
\hline Sodio $^{* *}$ & $147 \mathrm{mEq} / \mathrm{L}$ & \multicolumn{2}{|c|}{ Perfil lipídico } & TSH & $1,76 \mathrm{mU} / \mathrm{L}$ \\
\hline $\begin{array}{l}\text { Potasio } \\
\text { Cloro }\end{array}$ & $\begin{array}{l}3,7 \mathrm{mEq} / \mathrm{L} \\
109 \mathrm{mEq} / \mathrm{L}\end{array}$ & Prueba & Resultado & $\begin{array}{l}\text { T4L* } \\
\text { IGF1 }\end{array}$ & $\begin{array}{l}0,595 \mathrm{ng} / \mathrm{dl} \\
<15 \mathrm{ng} / \mathrm{ml}\end{array}$ \\
\hline Calcio & $8,7 \mathrm{mEq} / \mathrm{L}$ & Colesterol total ${ }^{\star \star}$ & $871 \mathrm{mg} / \mathrm{dl}$ & Prolactina & $46 \mathrm{ng} / \mathrm{ml}$ \\
\hline \multicolumn{2}{|c|}{ Uroanálisis } & & $\begin{array}{l}16 \mathrm{mg} / \mathrm{dl} \\
784 \mathrm{mg} / \mathrm{dl}\end{array}$ & $\begin{array}{l}\mathrm{FSH}^{*} \\
\mathrm{LH}^{*}\end{array}$ & $\begin{array}{l}0,1 \mathrm{mUl} / \mathrm{ml} \\
0,1 \mathrm{mUl} / \mathrm{ml}\end{array}$ \\
\hline Prueba & Resultado & Triglicéridos** & 169 mg/dl & Estradiol & $<10 \mathrm{pg} / \mathrm{ml}$ \\
\hline Densidad $^{*}$ & 1.005 & & & Cortisol $^{*}$ & $4,9 \mathrm{mcg} / \mathrm{dl}$ \\
\hline
\end{tabular}

INR: relación normalizada internacional; C3: componente 3 del complemento; C4: componente 4 del complemento; VSG: velocidad de sedimentación globular; PCR: proteína $C$ reactiva; ALT: alanina aminotransferasa; AST: aspartato aminotransferasa; GGT: gamma-glutamil transferasa; LDH: lactato deshidrogenasa; FA: fosfatasa alcalina; BUN: nitrógeno ureico; HDL: lipoproteína de alta densidad; LDL: lipoproteína de baja densidad; Ig: inmunoglobulina; HAV: virus de la hepatitis A; AgHBs: antígeno de superficie del virus de la hepatitis B; Anti-HCV: anticuerpos contra hepatitis C; RPR: reagina plasmática rápida; TSH: hormona estimulante de la tiroides; T4L: tiroxina libre; IGF1: factor de crecimiento insulínico de tipo 1; FSH: hormona foliculoestimulante; LH: hormona luteinizante.

* Valor disminuido

** Valor elevado 
Los estudios electrofisiológicos mostraron potenciales evocados auditivos anormales indicativos de hipoacusia neurosensorial leve izquierda y potenciales visuales normales con integridad de la vía retinocortical.

La resonancia magnética abdominal evidenció el compromiso multifocal del hígado, con lesiones sólidas de localización aleatoria, hipointensas en T1 e isointensas en T2, hipovasculares, con caída de la señal en secuencias fuera de fase y medidas entre 9 y $20 \mathrm{~mm}$. Había dilatación de las vías biliares intrahepática y extrahepática, y el colédoco tenía un diámetro de $7,5 \mathrm{~mm}$ (figura 1).

En la radiografía de miembros inferiores, se observó un festoneado endóstico focal en la diáfisis de la tibia derecha, pero sin reacción perióstica. En la resonancia magnética se apreció un patrón parcheado en la médula ósea de las extremidades inferiores, con lesiones focales hipointensas en T1 e hiperintensas en STIR, en las diáfisis tibiales y femorales, y en la zona metadiafisiaria proximal de la tibia izquierda; no se demostró compromiso de los tejidos blandos (figura 2).
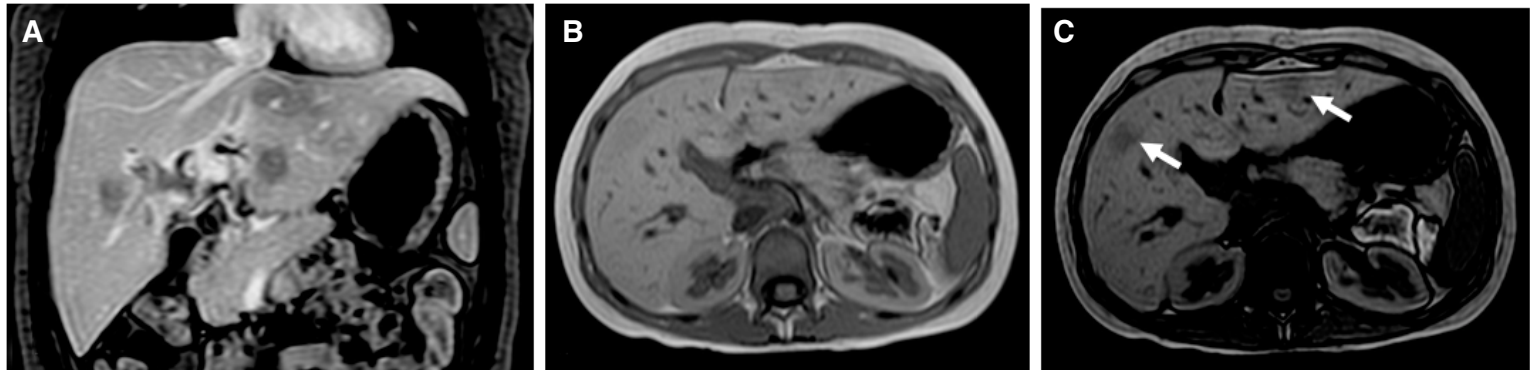

Figura 1. Resonancia magnética abdominal que evidencia lesiones hepáticas hipovasculares con contenido graso en T1 contrastado, con supresión grasa $(\mathbf{A})$, en fase $(\mathbf{B})$ y fuera de fase $(\mathbf{C})$
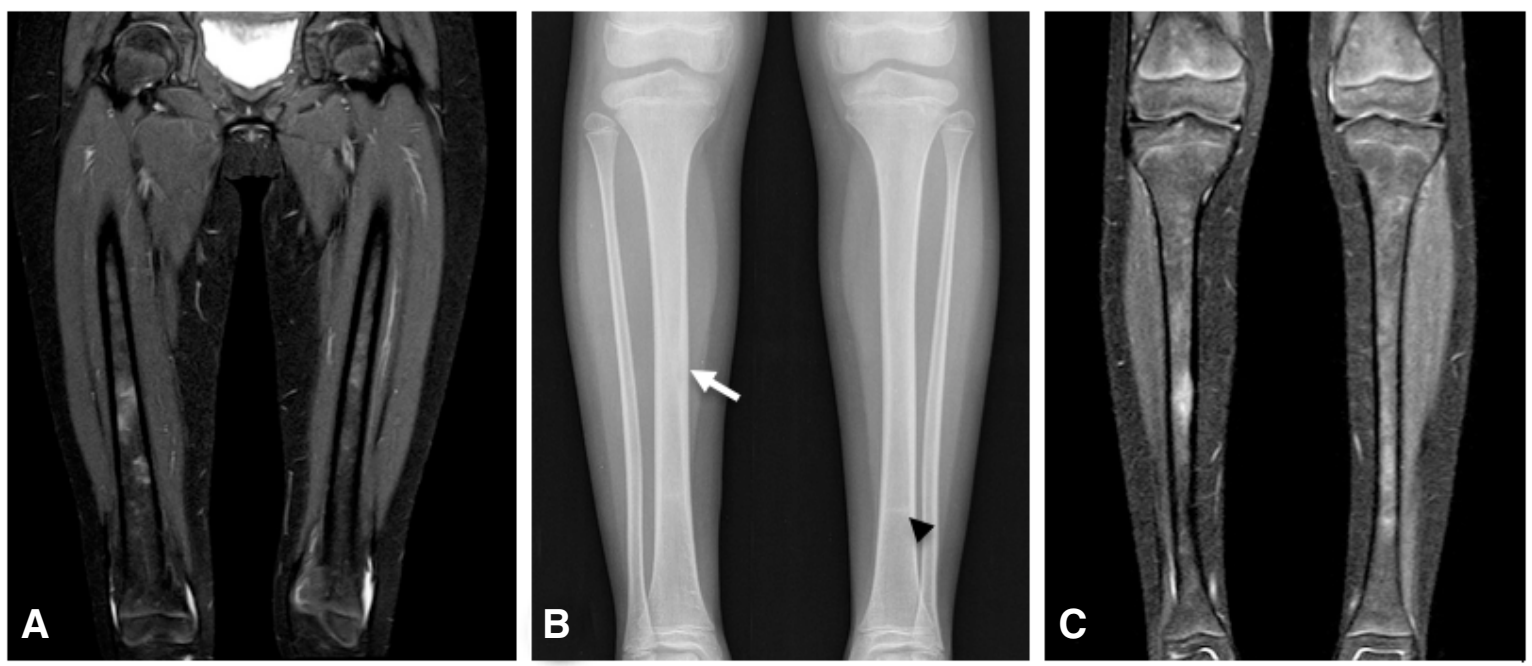

Figura 2. Extremidades inferiores. A. Resonancia magnética: engrosamiento cortical en el fémur. B. Radiografía simple: aspecto festoneado endóstico sin reacción perióstica (flecha) en las tibias y lesión esclerótica (punta de flecha) izquierda C. Resonancia magnética: patrón parcheado en la médula ósea, con lesiones focales hipointensas en T1 e hiperintensas en STIR, en las diáfisis tibiales y femorales, y en la zona metadiafisiaria proximal de la tibia izquierda. 
En la resonancia magnética cerebral se observaron dos masas extraxiales de bordes bien definidos, hipointensas en T1 y T2, con marcado realce. La primera masa era de localización selar y paraselar, con compromiso de los senos cavernosos, estenosis grave de las arterias carótidas internas y cerebrales posteriores, y compresión del quisma óptico y de la glándula pituitaria. La segunda masa era infratentorial derecha, con compresión del hemisferio cerebelar del mismo lado, pero sin efecto compresivo sobre el tallo cerebral ni el cuarto ventrículo. No se observó compromiso de los pares craneanos. Se evidenció engrosamiento de la duramadre en el tentorio y la hoz del cerebro, y además, dilatación e infiltración de los senos durales, el transverso derecho y la tórcula (figura 3).

Asimismo, se observó hipointensidad en las secuencias T2 en las regiones selar y paraselar, hallazgo correspondiente al signo oscuro o dark sign, es decir, baja intensidad de la señal, similar a la de la cortical ósea alrededor de la hipófisis y dentro de los senos cavernosos; este signo ha sido descrito en la hipofisitis autoinmunitaria y permite diferenciarla del adenoma hipofisario (10) (figura 4).
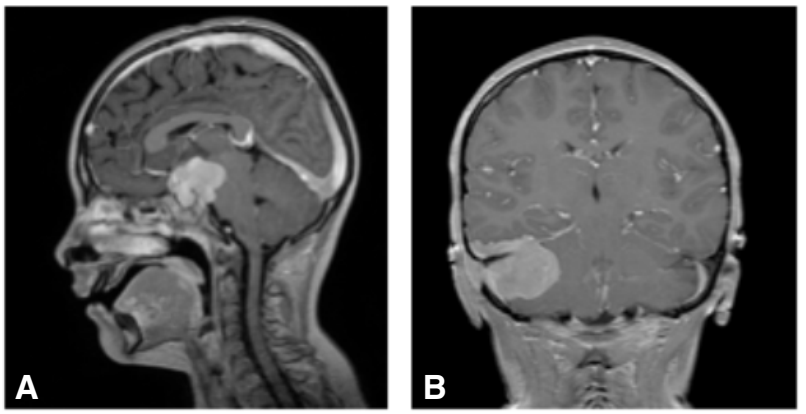

Figura 3. Resonancia magnética cerebral: $\mathbf{A}$. en plano sagital, lesión selar
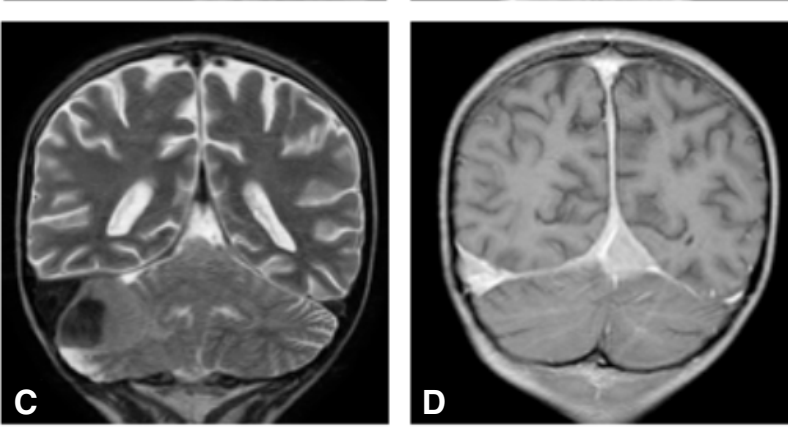
extraxial con marcado realce en la secuencia potenciada en T1 contrastado y aumento del realce de los senos durales; B. lesión infratentorial en plano coronal; C. la misma lesión con baja señal en T2 y aumento del tamaño del seno transverso derecho; $\mathbf{D}$. infiltración de la tórcula y el seno transverso derecho, así como engrosamiento dural bilateral en T1 contrastado.

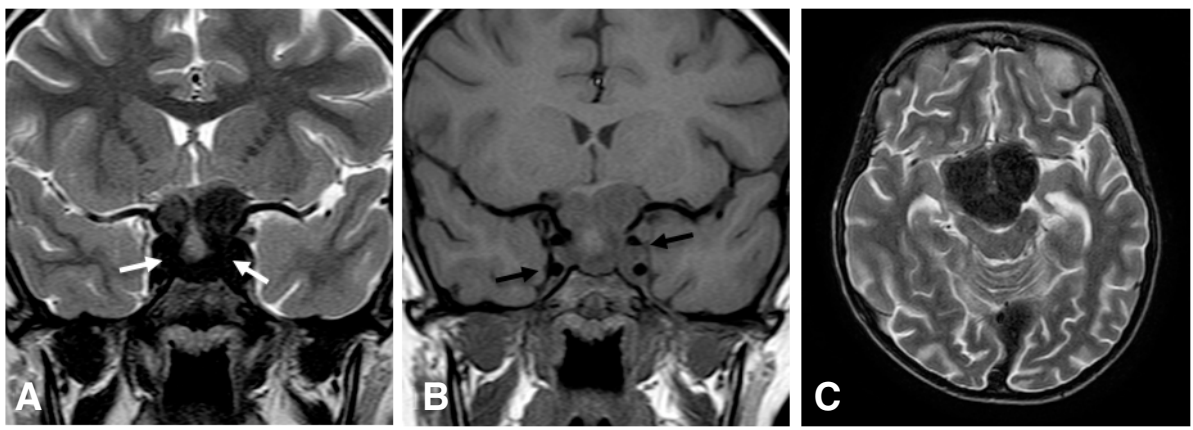

Figura 4. Resonancia magnética cerebral. A. el signo oscuro (flechas blancas) en secuencias potenciadas en T2 coronal y C. axial; B. segmento cavernoso de las arterias carótidas internas en T1 en el plano coronal (flechas negras) 

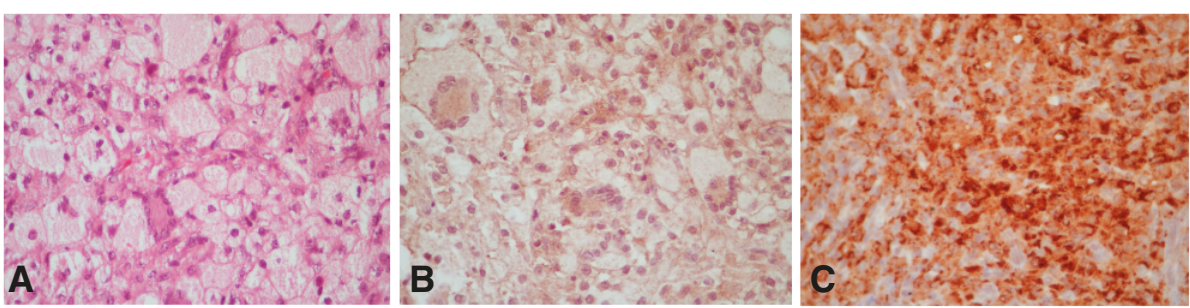

Figura 5. A. Duramadre infiltrada por histiocitos xantomatosos de tamaño variable, con células gigantes, algunas con múltiples núcleos que forman un anillo (células de Touton). Hematoxilina y eosina, 40X. B. Inmunohistoquímica con CD1a que muestra negatividad de las células xantomatosas con la tinción de membrana (expresión débil inespecífica para citoplasma), 40X. C. Inmunohistoquímica con CD68 que evidencia reactividad de las células xantomatosas, 40X.

Cuadro 2. Resultados de las pruebas genéticas y de inmunohistoquímica

\begin{tabular}{llll}
\hline Inmunohistoquímica & Genética & \\
\hline Prueba & Resultado & Prueba & Resultado \\
\hline CD68 & Acentuadamente positivo & Mutación BRAF V600E & Positiva heterocigota \\
S100 & Ocasionales células reactivas & & \\
CD1a & Negativo & & \\
CD45 & Positivo & & \\
Ki67 & Varios leucocitos en proliferación & & \\
\hline
\end{tabular}

En la biopsia intracraneal, se observó la infiltración por una lesión xantomatosa con histiocitos de tamaños variados mezclados con células de Touton, en músculo, hueso y duramadre (figura 5A). La inmunohistoquímica fue negativa para el patrón de membrana para CD1a, marcando en forma débil y heterogénea el citoplasma, y fue reactiva para CD68 (figura 5B-C). Algunas células mostraron reactividad variable (fuerte o débil) para el S100, por lo que se planteó que podría tratarse de una histiocitosis no Langerhans o una dislipidemia con xantomas asociados (figura 5).

Se practicó una biopsia hepática en la que se observó el hígado nodular con puentes fibrosos edematosos, proliferación de los conductos y abundantes macrófagos que predominaban en la interfase lesionando la placa limitante, con reacción positiva para CD68 y negativa para CD1a; no se observaron células xantomatosas ni de tipo Touton. Con la correlación histológica, se sugirió que podría tratarse de una enfermedad de ErdheimChester (cuadro 2).

En las pruebas genéticas, se encontró la mutación BRAF V600E, por lo que se inició la administración de $50 \mathrm{mg}$ de dabrafenib cada 12 horas por vía oral; inicialmente, la paciente toleró adecuadamente el tratamiento (cuadro 2).

\section{Consideraciones éticas}

Se obtuvo autorización escrita del acudiente de la paciente para usar la información clínica y las pruebas diagnósticas descritas en esta publicación.

\section{Discusión}

Para establecer la situación actual y los casos reportados de la enfermedad, se hizo una búsqueda sistemática en Medline (Ovid) y LILACS, tanto con lenguaje controlado como con texto libre (anexo 1). Se encontraron 66 referencias en las cuales se evaluaron el título, el resumen y el texto completo para seleccionar los reportes de caso o series de casos de la enfermedad de Erdheim-Chester en pacientes menores de 18 años: 19 artículos cumplían con los criterios de inclusión (7,9,11-31). Se excluyeron 
47, de los cuales cinco correspondían a series de casos de enfermedad de Erdheim-Chester que no discriminaban entre adultos y niños. El total de casos reportados fue de 22 (cuadro 3). Los estudios se habían desarrollado en Asia (11 casos), Europa (7 casos) y América (4 casos). La edad de los pacientes fluctuaba en un rango entre 1,7 y 17 años, con un promedio de 9 años; 11 casos fueron en niños y 11 en niñas. En todos los pacientes se estableció el compromiso óseo, seguido por el del sistema nervioso central en $55 \%$ de ellos. Se reportó también compromiso orbitario, cutáneo, abdominal, pulmonar, renal, pleural, mediastinal y retroperitoneal.

El diagnóstico se confirmó mediante histología e inmunohistoquímica en todos los casos. Por histopatología, se confirmaron 19 casos de enfermedad de Erdheim-Chester sola y tres de la mixta, de histiocitosis de células de Langerhans más enfermedad de Erdheim-Chester. Además, en siete de los casos se estudió la posible mutación V600E del gen $B R A F$, la cual se confirmó en cuatro (4/7, $57 \%)$ de ellos.

Cuadro 3. Características de los casos

\begin{tabular}{|c|c|c|c|c|c|c|c|c|c|c|c|}
\hline Año & País & $\begin{array}{l}\text { Edad } \\
\text { (años) }\end{array}$ & Sexo & $\begin{array}{l}\text { Extensión de la } \\
\text { enfermedad }\end{array}$ & Histología & BRAF & Tratamiento & Resp. & Seg. & Com. & Ref. \\
\hline 2019 & Colombia & 12 & $\mathrm{~F}$ & SNC, OSE, ABD & EEC & + & Dabrafenib & Sí & $1 \mathrm{~A}$ & Ninguna & RA \\
\hline 2018 & China & 11 & $M$ & SNC, OSE, ORB & HCL/EEC & + & Cirugía, dabrafenib & Sí & NR & NR & (9) \\
\hline 2018 & China & 3,5 & M & OSE, CUT & EEC & - & INT $\alpha / 2 \mathrm{a}$ & Sí & NR & NR & (12) \\
\hline 2017 & Hungría & 1,7 & $M$ & OSE, CUT & $\mathrm{HCL} / \mathrm{EEC}$ & + & Vemurafenib (3a línea) & Sí & $2 \mathrm{~A}$ & NR & (13) \\
\hline 2016 & India & 6 & $F$ & OSE & EEC & - & NR & NR & NR & LLA & (14) \\
\hline 2016 & EUA & 15 & M & SNC, OSE & EEC & NR & Anakinra, vinblastina & Sí & NR & NR & (15) \\
\hline 2016 & CdS & 3 & M & SNC, OSE, ORB & HCL/EEC & + & $\begin{array}{l}\text { Cladribina, citarabina } \\
\text { (2a línea) }\end{array}$ & Sí & NR & NR & $(16)$ \\
\hline 2016 & EUA & 7 & $\mathrm{M}$ & SNC, OSE & EEC & + & Anakinra (4a línea) & Sí & $2 \mathrm{~A}$ & NR & (17) \\
\hline 2015 & Irán & 14 & $\mathrm{M}$ & SNC, ORB & EEC & NR & Cirugía, citotóxico, CS & Sí & $8 \mathrm{~A}$ & NR & (18) \\
\hline 2014 & Singapur & 14 & $\mathrm{~F}$ & SNC, OSE & EEC & NR & INT $\alpha$ & Sí & $1 \mathrm{~A}$ & LLA, DM & (19) \\
\hline 2014 & México & 2 & $\mathrm{~F}$ & OSE, ORB & EEC & NR & $\begin{array}{l}\text { Vinblastina, CS (2a } \\
\text { línea) }\end{array}$ & Sí & $5 \mathrm{~A}$ & NR & (11) \\
\hline 2012 & China & 11 & $\mathrm{~F}$ & OSE & EEC & NR & INT $\alpha$ & Sí & $4 \mathrm{M}$ & NR & (20) \\
\hline 2012 & CdS & 4 & $M$ & SNC, OSE, PUL & EEC & NR & $\begin{array}{l}\text { INT } \alpha / 2 a \text {, ciclosporina, } \\
\text { CS (2a línea) }\end{array}$ & Sí & $6 \mathrm{M}$ & NR & (21) \\
\hline 2011 & China & 11 & $\mathrm{~F}$ & OSE & EEC & NR & $\begin{array}{l}\text { La familia rechaza el } \\
\text { tratamiento }\end{array}$ & NR & NR & NR & (22) \\
\hline 2010 & Francia & 17 & $M$ & OSE, ORB, REN & EEC & NR & NR & NR & NR & NR & (23) \\
\hline 2010 & Francia & 6 & $\mathrm{~F}$ & SNC, OSE, REN, PLE & EEC & NR & INT $\alpha$ & Sí & NR & NR & (23) \\
\hline 2009 & Francia & 10 & $\mathrm{~F}$ & OSE, RET & EEC & - & $\begin{array}{l}\text { Canakinumab (3a } \\
\text { línea) }\end{array}$ & Sí & $5 \mathrm{~A}$ & NR & $(24-26)$ \\
\hline 2007 & Turquía & 10 & $\mathrm{M}$ & SNC, OSE & EEC & NR & CS & NR & NR & NR & (27) \\
\hline 2005 & Japón & 13 & $\mathrm{~F}$ & SNC, OSE, ABD & EEC & NR & NR & NR & NR & NR & (28) \\
\hline 2004 & CdS & 10 & $\mathrm{~F}$ & OSE & EEC & NR & CS & Sí & $3 A$ & NR & $(29,30)$ \\
\hline 2003 & Italia & 14 & $\mathrm{~F}$ & SNC, OSE, ABD & EEC & NR & $\begin{array}{l}\text { Carboplatino, etopósido } \\
\text { (2a línea) }\end{array}$ & $+/-$ & $3 A$ & NR & (31) \\
\hline 1991 & EUA & 7 & M & SNC, OSE, MED, RET & EEC & NR & NR & NR & $2,5 A^{*}$ & NR & (7) \\
\hline 1991 & Francia & 17 & $\mathrm{~F}$ & OSE, ORB & EEC & NR & CS & NR & $2,5 A^{*}$ & NR & (7) \\
\hline
\end{tabular}

EUA: Estados Unidos de América; CdS: Corea del Sur; SNC: sistema nervioso central; OSE: óseo; ORB: orbitario; CUT: cutáneo; PUL: pulmonar; REN: renal; PLE: pleural; RET: retroperitoneal; ABD: abdominal; MED: mediastinal; BRAF: mutación BRAF V600E; NR: no reporta; RA: reporte actual; INT区: interferón alfa; CS: corticoesteroide; A: años; M: meses; LLA: leucemia linfoide aguda; DM: diabetes mellitus; Resp.: respuesta al tratamiento; Seg.: seguimiento; Com.: comorbilidades; Ref.: referencias; EEC: enfermedad de Erdheim-Chester; HCL/EEC: histiocitosis de células de Langerhans más enfermedad de Erdheim-Chester. 
El tratamiento se reportó en 17 de los casos y, en siete de estos pacientes, fue necesario utilizar más de un tipo de tratamiento. Los esquemas terapéuticos definitivos incluyeron: inmunosupresores como corticoesteroides en seis casos y ciclosporina en uno, interferón alfa en cinco, antagonistas del receptor de interleucina en uno, anakinra en dos y canakinumab en uno; además, se usó quimioterapia citotóxica en cinco casos, inhibidores de la enzima asociada al BRAF en dos y cirugía en otros dos. La respuesta al tratamiento se consideró adecuada en 14 de los 15 (93\%) pacientes en quienes se reportó, en tanto que en el otro caso la respuesta fue parcial. El seguimiento de los pacientes se describió en 12 casos, con una media de tiempo de 2,8 años. Se reportó la muerte de dos pacientes a los 2,5 años del diagnóstico de la enfermedad.

En el presente caso, se presentaron los dos tipos de compromiso más frecuentes en los pacientes pediátricos: el óseo, descrito en el $100 \%$ de los casos, y el del sistema nervioso central, descrito en el $55 \%$ de ellos. A diferencia de lo reportado en la literatura, en esta paciente la presentación clínica fue un síndrome ictérico debido al compromiso hepático confirmado histológicamente.

Las imágenes diagnósticas no solo fueron indispensables para detectar el compromiso óseo, esencial en el diagnóstico de la enfermedad de ErdheimChester, sino también, las lesiones hepáticas y cerebrales (infiltración de los senos durales), lo que contribuyó a la planeación y la toma de las biopsias. Además, se detectó el hallazgo radiológico denominado signo oscuro paraselar o dark sign, observado en las secuencias T2 (figura 2), el cual consiste en la baja intensidad de la señal alrededor de la hipófisis y dentro de los senos cavernosos que, hasta el momento, se ha descrito en pacientes con hipofisitis autoinmunitaria para diferenciarlos de aquellos con adenomas hipofisarios (10).

Este es el primer caso de enfermedad de Erdheim-Chester en la población pediátrica reportado en Colombia y el segundo en Latinoamérica. En Colombia, se había reportado un caso de esta enfermedad en un adulto de 53 años con diagnóstico de fractura patológica de húmero (32). En Latinoamérica, se había reportado un caso en México, el de una niña de dos años de edad con compromiso óseo y orbitario, cuya reacción terapéutica a la quimioterapia citotóxica de segunda línea con vinblastina y corticoesteroides fue adecuada (11).

\section{Conclusión}

La enfermedad de Erdheim-Chester es una condición extremadamente rara en la infancia, cuya presentación es heterogénea y multisistémica. Para lograr un diagnóstico adecuado, se deben tener en cuenta los hallazgos clínicos, los histopatológicos y los radiológicos. Los reportes de casos de la enfermedad permiten conocerla mejor $y$, por ende, diagnosticarla y tratarla de forma adecuada.

\section{Referencias}

1. Emile JF, Abla O, Fraitag S, Horne A, Haroche J, Donadieu J, et al. Revised classification of histiocytoses and neoplasms of the macrophage-dendritic cell lineages. Blood. 2016;127:2672-81. https://doi.org/10.1182/blood-2016-01-690636

2. Chester W. Über lipoidgranulomatose. Virchows Arch Pathol Anat. 1930;279:561-602. https://doi.org/10.1007/BF01942684 
3. The Writing Group of the Histiocyte Society. Histiocytosis syndromes in children. Lancet. 1987;329:208-9. https://doi.org/10.1016/S0140-6736(87)90016-X

4. Diamond EL, Dagna L, Hyman DM, Cavalli G, Janku F, Estrada-Veras J, et al. Consensus guidelines for the diagnosis and clinical management of Erdheim-Chester disease. Blood. 2014;124:483-92. https://doi.org/10.1182/blood-2014-03-561381

5. Rascón-Ramírez FJ, Avecillas-Chasín JM, Rodríguez-Boto G, Subhi-Issa I, Salazar OA, Sallabanda K. Enfermedad de Erdheim-Chester, una gran imitadora. Presentación de casos y revisión de la literatura. Neurocirugía. 2016;27:296-303.

6. Ozkaya N, Rosenblum MK, Durham BH, Pichardo JD, Abdel-Wahab O, Hameed MR, et al. The histopathology of Erdheim-Chester disease: A comprehensive review of a molecularly characterized cohort. Mod Pathol. 2018;31:581-97. https://doi.org/10.1038/modpathol.2017.160

7. Veyssier-Belot C, Cacoub P, Caparros-Lefebvre D, Wechsler J, Brun B, Remy M, et al. Erdheim-Chester disease. Clinical and radiologic characteristics of 59 cases. Medicine (Baltimore). 1996;75:157-69. https://doi.org/10.1097/00005792-199605000-00005

8. Mazor RD, Manevich-Mazor M, Shoenfeld Y. Erdheim-Chester disease: A comprehensive review of the literature. Orphanet J Rare Dis. 2013;8:137. https://doi.org/10.1186/1750-1172-8-137

9. Hao X, Feng R, Bi Y, Liu Y, Li C, Lu T, et al. Dramatic efficacy of dabrafenib in ErdheimChester disease (ECD): A pediatric patient with multiple large intracranial ECD lesions hidden by refractory Langerhans cell histiocytosis. J Neurosurg Pediatr. 2018;23:48-53. https://doi.org/10.3171/2018.6.PEDS17728

10. Nakata $\mathrm{Y}$, Sato $\mathrm{N}$, Masumoto $\mathrm{T}$, Mori $\mathrm{H}$, Akai $\mathrm{H}$, Nobusawa $\mathrm{H}$, et al. Parasellar T2 dark sign on MR imaging in patients with lymphocytic hypophysitis. AJNR Am J Neuroradiol. 2010;31:1944-50. https://doi.org/10.3174/ajnr.A2201

11. Quero-Hernández A, Echeverría R, Zárate A, Escárraga V, Garzón S, Reyes M, et al. Enfermedad de Erdheim-Chester en un niño con lisis ósea simétrica. Rev Mex Pediatr. 2014;81:222-5

12. Su HH, Wu W, Guo Y, Chen HD, Shan SJ. Paediatric Erdheim-Chester disease with aggressive skin manifestations. Br J Dermatol. 2018;178:261-4. https://doi.org/10.1111/bjd.15306

13. Váradi Z, Bánusz R, Csomor J, Kállay K, Varga E, Kertész G, et al. Effective BRAF inhibitor vemurafenib therapy in a 2-year-old patient with sequentially diagnosed Langerhans cell histiocytosis and Erdheim-Chester disease. Onco Targets Ther. 2017;10:521-6. https://doi.org/10.2147/OTT.S121615

14. Vallonthaiel AG, Mridha AR, Gamanagatti S, Jana M, Sharma MC, Khan SA, et al. Unusual presentation of Erdheim-Chester disease in a child with acute lymphoblastic leukemia. World J Radiol. 2016;8:757-63. https://doi.org/10.4329/wjr.v8.i8.757

15. White TV, Silvester NC, Otero HJ. Non-sclerotic bone involvement in Erdheim-Chester: PET/ CT and MRI findings in a 15-year-old boy. Pediatr Radiol. 2016;46:1345-9. https://doi.org/10.1007/s00247-016-3594-y

16. Kim S, Lee M, Shin HJ, Lee J, Suh Y. Coexistence of intracranial Langerhans cell histiocytosis and Erdheim-Chester disease in a pediatric patient: A case report. Child's Nerv Syst. 2016;32:893-6. https://doi.org/10.1007/s00381-015-2929-6

17. Diamond EL, Abdel-Wahab O, Durham BH, Dogan A, Ozkaya N, Brody L, et al. Anakinra as efficacious therapy for 2 cases of intracranial Erdheim-Chester disease. Blood. 2016;128:1896-8. https://doi.org/10.1182/blood-2016-06-725143

18. Alimohamadi M, Hartmann C, Paterno V, Samii M. Erdheim-Chester disease mimicking an intracranial trigeminal schwannoma: Case report. J Neurosurg Pediatr. 2015;15:493-8. https://doi.org/10.3171/2014.10.PEDS14441

19. Krishna VV, James TE, Chang KT, Yen SS. Erdheim-Chester disease with rare radiological features in a 14-years old girl with pre-B acute lymphocytic leukemia and diabetes mellitus. J Radiol Case Rep. 2014;8:7-15. https://doi.org/10.3941/jrcr.v8i8.1899

20. Wen C, Liang Q, Yi Z, Wan W. Erdheim-Chester disease in a child. West Indian Medical J. 2012;61:834-7. https://doi.org/10.7727/wimj.2012.161

21. Song SY, Lee SW, Ryu K, Sung SH. Erdheim-Chester disease with multisystem involvement in a 4-year-old. Pediatr Radiol. 2012;42:632-5. https://doi.org/10.1007/s00247-011-2235-8 
22. Wen C, Liang Q, Wan W. Erdheim-Chester disease in a child: Case report. Chinese J Pediatr. 2011;49:231-2. https://doi.org/10.3760/cma.j.issn.0578-1310.2011.03.017

23. Arnaud L, Pierre I, Beigelman-Aubry C, Capron F, Brun AL, Rigolet A, et al. Pulmonary involvement in Erdheim-Chester disease: A single-center study of thirty-four patients and a review of the literature. Arthritis Rheum. 2010;62:3504-12. https://doi.org/10.1002/art.27672

24. Tran TA, Pariente D, Guitton C, Delwail A, Barat-Houari M, Meinzer U. Treatment of ErdheimChester disease with canakinumab. Rheumatology. 2014;53:2312-4.

https://doi.org/10.1093/rheumatology/keu344

25. Tran TA, Pariente D, Lecron JC, Delwail A, Taoufik Y, Meinzer U. Treatment of pediatric Erdheim-Chester disease with interleukin-1-targeting drugs. Arthritis Rheum. 2011;63:40312. https://doi.org/10.1002/art.30638

26. Tran TA, Fabre M, Pariente D, Craiu I, Haroche J, Charlotte F, et al. Erdheim-Chester disease in childhood: A challenging diagnosis and treatment. J Pediatr Hematol Oncol. 2009;31:7826. https://doi.org/10.1097/MPH.0b013e3181b76827

27. Kumandaş S, Kurtsoy A, Canöz Ö, Patıroğlu T, Yikilmaz A, Per H. Erdheim-Chester disease: Cerebral involvement in childhood. Brain Dev. 2007;29:227-30.

https://doi.org/10.1016/j.braindev.2006.08.013

28. Nagatsuka H, Han PP, Taguchi K, Tsujigiwa H, Gunduz M, Fukunaga J, et al. ErdheimChester disease in a child presenting with multiple jaw lesions. J Oral Pathol Med. 2005;34:420-2. https://doi.org/10.1111/j.1600-0714.2005.00320.x

29. Sohn MH, Kim MW, Kang YH, Jeong HJ. Tc-99m MDP bone and Ga-67 citrate scintigraphy of Erdheim-Chester disease in a child. Clin Nucl Med. 2006;31:90-2. https://doi.org/10.1097/01.rlu.0000196413.47370.c9

30. Joo CU, Go YS, Kim IH, Kim CS, Lee SY. Erdheim-Chester disease in a child with MR imaging showing regression of marrow changes. Skeletal Radiol. 2005;34:299-302. https://doi.org/10.1007/s00256-004-0846-y

31. Clerico A, Ragni G, Cappelli C, Schiavetti A, Gonfiantini M, Uccini S. Erdheim-Chester disease in a child. Med Pediatr Oncol. 2003;41:575-7. https://doi.org/10.1002/mpo.10402

32. Gómez L, Soto C, Criollo F, Messa Ó, Romo R, Patrón I. Enfermedad de Erdheim-Chester: fractura patológica de húmero izquierdo. Rev Colomb Cancerol. 2014;18:143-8. https://doi.org/10.1016/j.rcc.2014.05.002 